\title{
URTICARIA TUBEROSA OF WILLAN
}

\author{
By J. A. NIXON \\ Historical Survey.
}

The term 'urticaria tuberosa' was first used by Frank (1) in his work De Curandis Hominum Morbis, 1792. In his account of the exanthemata he applied the term to a large group which now would include the manifold varieties of angeio-neurotic oedema. His definition of urticaria tuberosa reads thus: 'A definition of this exanthem is, from its inconsistency, difficult. We shall not form a bad idea of the disease if we describe it as an efflorescence, often but not always accompanied by fever, consisting sometimes of macules like nettle-stings, sometimes of tumours surmounted by a vesicle, or even of great protuberances in the skin. It usually begins towards night with intense itching of the skin and a slight fever, or it may break out profusely upon exposure of the skin to cold, itching intolerably. The rash disappears readily but soon breaks out again with scratching, but after a few days it usually subsides without desquamation of the epidermis.' Frank gives a detailed description of a young man, aged 20, affected by this form of urticaria, which seems indistinguishable from any other form of food urticaria attended by gastro-intestinal disturbance and a profuse nettle-rash.

Willan (2), however, in 1808, distinguished this variety of urticaria rather more clearly than Frank from the general group. 'In the urticaria tuberosa', he says, 'many of the wheals increase to a large size, forming hard tuberosities, which seem to extend deeply into the muscular flesh, and occasion a contraction in the sinews, with total inability of motion, and a sensation of pain in the bones. These tumours are usually whitish at their tops: they rise on the arms, thighs, loins, and calf of the leg, and are very hot and painful for several hours. The exuption, in all cases under my obscrvation, took place at night, and before morning it wholly disappeared, leaving the patient weak, languid, and sore, as if he had been bruised, or had undergone much fatigue.'

Cazenave (3), 1827, gives a good account which Rayer follows very closely of a case observed in Biett's clinic. Bateman (4), 1829, gives his description in Willan's words, prefacing them with the remark: 'This species which was named by Dr. Frank.' Rayer (5), 1835, is the next to refer to this variety of urticaria : 'Chronic urticaria, however, is sometimes seen with more serious characters (urticaria tuberosa of Willan). The disease does not then consist in mere slightly prominent elevations, but in true tuberosities of various magnitudes, hard, deep-seated, extending to the subcutaneous cellular tissue, sometimes accompanied by true ecchymoses, by pain in moving the limbs, and a tense very sore state of the skin. These tumours, which are very itchy, appear in the evening or at night, and disappear again entirely before morning, leaving the patient weak, restless, 
and weighed down with languor and depression. They come out more particularly on the loins and extremities, but they may show themselves over the whole of the body, cause a general swelling of the face, of the neck, and of the limbs; be accompanied by dyspnoea, irregularity in the action of the heart and other symptoms distressing in various degrees which are commonly developed under the influence of a febrile paroxysm (febris intermittens urticata, Frank). The eruption disappears completely with the remission of the fever and appears withits accession.'

After this time, the variety named by Willan, urticaria tuberosa, seems to have been lost sight of. Demarquay (6) in 1842, described the treatment of several cases of urticaria tuberosa, but this is an article in praise of arsenic rather than a particularly careful account of the conditions treated. He quotes indeed Frank and Bateman, and at greater length Rayer and Cazenave, but his cases include one of acute circumscribed oedema with hydrarthrosis and a septic case with enormous painful swelling of the limbs and ulceration of the skin.

Graves (7), 1843, came nearer to the truth when in his 'Clinical Lectures' he wrote: 'You will allow that the connexion between arthritis, disease of the digestive organs and urticaria can no longer be considered as fortuitous.' Speyer (8) in 1853 published an article entitled ' Urticaria Tuberosa Frankii' in which a general description of urticaria is given without attention to the distinctive features upon which Willan based his classification. Fouquet(9), 1865, gives the most complete survey of the early references to urticaria tuberosa that I have been able to find. Although he credits Frank with having first used the name, he is inclined to consider the single case which Frank recorded as being a doubtful instance of the disease. Fouquet comments on the extreme rarity of the condition, and the very few examples which had to that time been reported. He rejects Fuch's three cases recorded under this title, as well as Biett's case described by Cazenave, which is, as he says, quoted at length in most books on dermatology, although, being unique, it cannot properly be regarded as a confipmation or illustration of this variety of urticaria. Fouquet suggests that the condition called by Frank 'urticaria tuberosa' very soon lost its identity in Bateman's 'erythema nodosum'. He does not, however, mention that the disease as described by himself was first completely differentiated by Willan under the title ' urticaria nodosa seu tuberosa', nor does he seem to appreciate the close similarity between his own description and Willan's.

Fouquet regarded the disease as peculiar to the female sex, occurring usually between the years of puberty and the climacteric, and generally associated in some way with the uterine functions. There is, as a rule, no prodromal stage. An eruption suddenly appears consisting of a few rapidly developing wheals which change incredibly quickly into hard, sharply defined nodes or knobs of round, oval or irregular shape, sometimes attaining on the loins to the size of the hand. These elevations are white, with occasionally a slight erysipelatous reddening around. The eruption is accompanied by a burning pain which renders movement difficult. The tuberosities are apt to appear at nights and disappear by the morning (epinyctides). The head and face are not affected, the 
farourite sites being the lower limbs and loins. The tuberosities are hard, freely movable, and do not itch, but cause an intolerable burning or aching pain. They are deeply seated in the true skin, but Fouquet does not accept the statement of some authors that they involve the subcutaneous cellular tissue. He lays special stress on their ephemeral nature; there are rarely more than two or three tuberosities present at one time, they disappear as a rule within twenty-four hours, and a fresh crop will break out every night. This tendency to recurrence may last from a fortnight up to several months, with increasing periods of freedom, and a diminution in the size of the nodes. The patients feel weak, exhausted, and sore, as if their limbs had been beaten. Fouquet describes five cases with almost identical symptoms occurring in women of child-bearing age varying from about 30 to 50 . He admits that the immediate causation is absolutely unknown, and that the treatment is unsatisfactory. Cold and exposure to wind and weather may precipitate an attack, while warmth relieves the pain. Heating food or drink aggravates the condition. The preference for the female sex and some relation to disturbed sexual functions he regards as indisputable.

Morrant Baker (10), 1881, once again made use of the term to describe an ordinary case of superficial urticaria complicated by Bazin's erythema induratum.

A new era, however, commenced with Quincke's masterly description of acute circumscribed oedema in 1882. Beyond acknowledging the merits of Quincke's observations there is small need to recapitulate his conclusions, which tended to direct attention away from Willan's urticaria tuberosa. So that since this period, urticaria tuberosa appears to have been lost sight of.

Although pathogenetically the condition is allied to Quincke's acute circumscribed oedema, the patients subject to the diseasc are not diagnosed as suffering from an affection of the kind described by Quincke. So much is this the case, that, after the appearance of acute circumscribed oedema in our nomenclature, the term urticaria tuberosa vanished. This would be a matter of small moment if the condition were still recognized under Quincke's classification as an oedema or an urticaria. In point of fact, however, the cases have been relegated to the lumber heap of chronic rheumatisms and discouraging prognoses accordingly given to the sufferers.

Dubreuilh (11), 1892, objected to the name acute circumscribed oedema, and suggested as an improvement 'urticaire cedémateuse', but he describes a form of urticaria in which nodules occur without pyrexia or local heat. The nodules are very hard, and scarcely red. Itching is slight or absent, but there is a disagreeable sense of tension. He gives an excellent description of this nodular urticaria in the fingers, where 'if the swelling is not obvious to the eye, it is very clearly perceived by the patient, who feels his fingers so swollen that it is painful to bend them'.

Collins (12), 1892, temporarily replaced the condition in its right category, when in his description of angeio-neurotic oedema he gives as synonymous titles 'acute circumseribed oedema, acute idiopathic oedema, periodic swelling, urticaria tuberosa or giant swelling, acute non-inflammatory oodema, 
Australian blight'. He uses the term 'urticaria tuberosa' as the equivalent of the whole group of angeio-neurotic oedemas, and not in the more restricted sense of Willan. But he makes the important observation: 'this condition has a close relationship to the many oedemas spoken of, and also a family relation with many of the arthropathies as yet not well understood, but known to be directly caused through the agency of the nervous system.' Osler (13), 1909, refers to this type of urticaria very briefly: 'a peri-articular variety has been described.'

Garrod (14), 1907, admits 'that there are good reasons for thinking that such a transient arthritis as that of rbeumatic fever has a close kinship with such inflammatory forms of oedema as are met with in the erythemas and urticaria, and the frequency of their clinical association strengthens this belief; but one cannot help feeling that some writers have laid undue stress upon the presence, in one or two recorded cases of the articular trouble, of symptoms which appear to be frequently associated with acute circumscribed oedema'.

I think to this group belong those cases referred to by F. E. Batten (15), 1910, as 'polymyositis in association with erythema nodosum and urticaria'. 'In erythema nodosum it is not uncommon to find the underlying muscles hard and tender to pressure; but as the erythema nodosum clears up the affection of the muscles passes off; in some reported cases, however, the affection of the muscles is by no means so transient. The cause of this form of myositis is obscure: cultures made from muscles gave negative results, but the association with erythema nodosum and urticaria would indicate that the poison is probably of intestinal origin.' Batten describes the symptoms as commonly affecting the lower extremities and in slighter forms coming on suddenly with severe urticaria of the skin and swelling of the muscles. The swollen muscles are painful, and any movement active or passive is acutely painful. In severer forms the lower extremities are considerably swollen and small indurated areas can be felt in the muscles. Rapid recovery is the rule, the acute symptoms pass off in a week, but some muscular tenderness may persist for a time. 'This form is prone to relapse.'

But it is in MeCall Anderson's (16), 1887, account that Willan's urticaria tuberosa meets with the first complete recognition since the time of Rayer. 'There is a variety of nettle-rash to which the term urticaria nodosa seu tuberosa has been given. It is a rare affection; it appears in the shape of pretty hard nodosities about the size of a split marble and of a reddish colour, which occur oftenest at night and disappear in a few hours, frequently recurring. They may involve any part, but the extremities and limbs are specially liable. In very rare cases they are associated with congestion, rupture of capillaries, and ecchymoses.'

Symptomatology.

The observation of McCall Anderson and Fouquet that this is a rare variety of urticaria appears to be correct. Since Willan's original description few cases are met with in medical literature. It would be of little importance if the conditions were common, easily recognized, and therefore considered too trivial to be reported. But the matter is not thus lightly to be dismissed. Although 
the disease may occur more commonly than the recorded instances would suggest, the cases appear to be obscure in nature and, for the most part, to be wrongly diagnosed. Perhaps their resemblance to rheumatoid arthritis leads to their being placed in this protean group of diseases rather than amongst the urticarias where they rightly belong.

The clinical picture of urticaria tuberosa is, however, not without distinctive features. Age affects its incidence little if at all. The sex distribution shows that in common with other forms of angeio-neurotic oedema females are somewhat more prone to be affected. A characteristic attack is marked by the sudden rapid development of subcutaneous or deep-seated swellings, varying in size from nodules as large as peas to tumours as big as oranges. They are multiple and distributed about the extremities, especially the fingers, hands, wrists, feet, and knees, occasionally about the loins and the back of the shoulders. These nodules rarely involve the skin, which, as a rule, shows no discoloration, but they appear to select fascia, tendon sheaths, and peri-articular tissues. The swellings are unaccompanied by a true cutaneous nettle-rash. They are hard, or, if large, may give rise to the sensation of deep-seated tense fluid swellings, pitting subcutaneously on prolonged pressure. Movement of the limbs involved may be stiff and painful. Sometimes the fingers are the seat of diffuse swelling, not situated at the joints but between them, giving rise to a fusiform appearance which resembles at first sight rheumatoid arthritis, but distinguishable therefrom by the presence of an apparent constriction over each joint.

The subjective sensations complained of, in addition to aching pain and stiffness, are tingling and burning but rarely itching. The nodules usually occur at night and disappear before morning, or they may be even more evanescent, lasting only a few hours. 'The patient is left weak, languid, heavy from want of sleep, and tired as if bruised. As a rule, there is no pyrexia and the pulse-rate is not increased.

Bearing in mind that the condition is commonly diagnosed as rheumatoid arthritis or some form of chronic rheumatism, it is important to note that there is no tendency to permanent damage of the joints. There is no grating or creaking to be felt, and no rarefaction, erosion, or osteophytic growths are discovered by X-rays. The joints do not become fixed, or deformed, and in the hands no deflexion of the phalanges results even from repeated attacks.

The patients are healthy and well-nourished, ready and able to excrcise as soon as the swellings have disappeared. Sometimes, indeed, exercise seems to disperse the swellings and remove the sensation of stiffness. Rubbing and even vigorous massage may actually conduce to a feeling of ease and comfort. Distension of the joint capsule with fluid does not seem to occur, al though bursal effusions may be present.

Cardiac complications are conspicuously absent, so too are organic nerve lesions and wasting of muscles.

The distribution of the swellings is not symmetrical, nor does it correspond with nerve or segmental areas. 
No urinary or menstrual irregularities coexist.

The blood examination reveals no abnormal cell-counts and cultures from the blood proved sterile; but there appears to be a definite delay in the coagulation-time of the blood during the attacks.

\section{Complications and Sequelae.}

No complications or sequelae have been observed. The condition seems to clear up suddenly and completely.

Gastro-intestinal disturbances, such as vomiting, colic, or diarrhoea, not infrequently precede or usher in an attack. This is the one occasional accompaniment which calls for attention.

\section{Aetiology.}

From the preceding remarks there is some probability that among the causes of urticaria tuberosa digestive derangements have to be reckoned with. Perhaps the victims have an idiosyncrasy to some particular article of diet whose identity cannot be traced and need not be the same for each individual. Or it may be that any interference with digestion leads to absorption of intestinal toxins which determine the outbreak. Exposure to cold and excessive exercise act as exciting causes, especially exposure to cold after being heated by muscular exertion. Heredity cannot be definitely established as a cause. Emotion seems to play no part. Illnesses of a debilitating nature, such as influenza and diphtheria, may in some degree account for the liability to attacks. Neuroses in a general sense are not observed in the patients.

\section{Prognosis.}

The prognosis may be summed up in a very few words.

Rapid recovery from an attack is the rule.

Recurrence is probable, and the liability to recurrence extends over many years, perhaps a lifetime.

Permanent injury does not occur.

\section{Diagnosis.}

The disease has to be distinguished from acute rheumatism, especially the modern variety in children. This should not be difficult. Pyrexia is either absent or very slight and transient. The patients cannot be described as ill, they are merely uncomfortable, unless the gastro-intestinal disturbance is very severe. The joints are really free from effusion. The heart remains unaffected. The nodules are not situated over bony prominences, as, for instance, the exposed border of the ulna.

From rheumatoid or osteo-arthritis, urticaria tuberosa may be differentiated by the fact that there is no permanent fixation of the joints and rarely any effusion into them. There is an absence of creaking, grating, or lipping of the articular end of the bones. X-rays show the joints to be normal in appearance. 
The patients are not wasted or pigmented, and there is no excessive sweating of the hands. On the contrary, the skin may show a remarkable absence of perspiration. There is no ulnar deflexion or flexion of the fingers. A curious hyper-extension of the phalanges has been noticed. Heberden's and Haygarth's nodes do not occur.

From gout the diagnosis is easy. The history of the attacks presents no features of resemblance. The exquisite pain and tenderness are lacking, and the skin over the affected joints does not assume any dusky redness. Tophi are not present.

\section{Description of Cases.}

Case I. Miss L., aged 50, a lady of independent means, who had resided for many years in Australia, was seen in consultation with Dr. A. L. Flemming in June, 1909. For many years she had suffered from attacks of rheumatism, which had recently been pronounced to be rheumatoid arthritis. She had visited Harrogate and other spas considered to be useful in rheumatic affections. Inquiry elicited the fact that she had on previous occasions suffered from ordinary attacks of urticaria (nettle-rash). The patient considered that exposure to cold was the chief exciting cause of the condition, and could not definitely associate its appearance with any other circumstances. Her general health and nutrition were good. The disease manifested itself by the sudden rapid appearance of hard swellings in various parts of the body, especially upon the fingers, wrists, deltoid, and rhomboid regions. One favourite spot she pointed out over the latissimus dorsi, which she used to ask her maid to "pummel as hard as she could, and it would go'. The tumours usually began at night, they might be present on getting up in the morning, but they were of a very fleeting nature and would often have disappeared by the time her doctor came to see her. Exercise seemed to lead to their disappearance. The particular attack in which I saw her was characterized by a large protuberance over the back of the right wrist, which at first sight presented the appearance of a dislocation of the wrist with the bones of the forearm prominent above the dorsum of the hand. This swelling was hard, tense, and deep-seated; it did not involve the skin completely, which was not, however, freely movable over the swelling. The skin was not discoloured and did not pit on pressure; but on prolonged firm pressure a deepseated subcutaneous pitting could be produced. The discornfort complained of was a dull aching with stiffness. The wrist-joint, however, could be freely moved without any sensation of creaking or grating. There was no effusion into the joint itself. The movements of the extensor tendons of the fingers were not limited, although the patient said they felt stiff and a slight creaking could

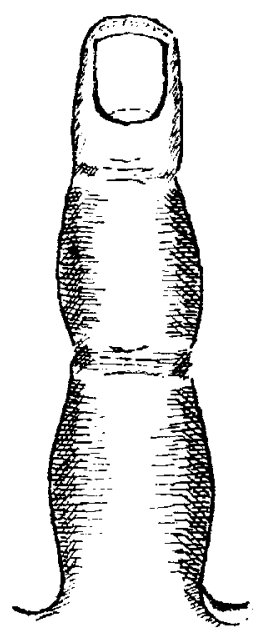

Spindle-shaped swelling of fingers. be detected suggestive of teno-synovitis. The fingers of both hands presented a curious spindle-shaped deformity, which at first sight resembled rheumatoid arthritis, until it was noted that the maximum swelling of each spindle corresponded with the centre of the phalanx and a constriction occurred at each inter-phalangeal joint. There were several discrete deep-seated nodules about the size of peas, not situated, like Heberden's nodes, at the joints and about the articular ends of the phalanges, but in the length of the phalangeal shaft. These 
nodules ached, but were not painful or tender to the touch. They were not in the skin, nor did they move with the tendons, but seemed to be situated on, rather than in, the tendon sheaths. Movement of the fingers was limited to a slight extent by stiffness, but there was no creaking or grating in the joints or tendon sheaths, and there was no fixation, flexion, or deviation of the fingers, and no lipping or osteophytic formation about the joints. Similar nodules, varying from the size of a pea to a walnut, were present in the rhomboids and deltoids. These felt precisely like those met with in nodular rheumatism, except that many of them were of a larger size. The patient complained of aching and stiffness in these regions as well as in the loins and hips, where no swellings could be detected.

A few hours later in the day Dr. Flemming again saw the patient and found the hand quite normal in appearance, the whole of the swellings having vanished. There was a feeling of slight malaise during and after the attack, and the patient said she felt as if she had been beaten all over. There had been a complete absence of the itching and burning of the skin usually associated with urticaria. No gastro-intestinal symptoms were present. The patient had passed the climacteric several years previously and suffered no pelvic disorders. She was aware that she had been subject to these attacks since childhood, and expressed her surprise that, contrary to the prognostications of many physicians, she was developing no permanent deformity of her joints, and between the attacks which came on at intervals, varying from a few days to many months, she enjoyed the free use of her limbs. In the course of discussion as to the nature of her malady she ventured the suggestion that she was the victim of "Australian blight", a name then quite unfamiliar to me and of whose exact nature I am still ignorant, save that it is given in a few books as a synonym of urticaria tuberosa. A blood-count showed no abnormal conditions. The coagulation time of her blood taken the following day showed a very slight delay.

The urine examination revealed nothing abnormal, and there was no evidence of renal disease or arterio-sclerosis.

A course of treatment with calcium lactate marked a complete cessation of symptoms, but such an interval of freedom from the attacks was not unusual apart from treatment.

Subsequent observation of this patient has shown that she occasionally develops spontaneous ecchymoses in the skin.

Case II. Miss A. C., aged 40, in 1908 suffered from a severe attack of influenza, which was followed by a condition called 'neuritis' in the arms and hands, unattended, however, by wasting or motor weakness. The symptoms complained of were intense pain shooting down the arms to the finger-tips, with tingling and stiffiness of the fingers; the fingers felt cold and dead; used to turn very white and swollen. The attacks were singularly transient and did not prevent her from playing croquet assiduously. This continued for three years, but I did not chance to see her during an attack, until, in May 1911, she showed me her hands with the fingers white and swollen, presenting a series of characteristic spindle-shaped swellings over the phalanges, and constrictions at the joints. In the flexor sheaths of the fingers were small hard nodules, which caused aching and stiffness, but without tenderness or pain on being handled. The joints were free from swelling; there was no creaking, grating, or fixation. No other parts of the body were affected.

Miss C. told me that this was the condition which during the last three years had been variously diagnosed as neuritis and rheumatism, and for which she had visited Continental and English health resorts famed for curing rheumatic diseases. The attacks were always very transient and as a rule began at night, disappearing during the first few hours of the morning after she got up. She thought moderate exercise and moving of the limbs cleared up the swelling. The tingling pain before the swellings developed was sometimes intense. Her opinion as to 
the causation was that exposure to cold and excessive exercise brought on the attacks. She was particularly liable to them after playing croquet during a hot summer afternoon and continuing until the colder evening drew on. Her general health was excellent; the heart, lungs, urine, and menstrual functions were normal.

She had never suffered from a true urticaria (nettle-rash), but about a year previously had developed two streaks of sclerodermia (morphoea) down the subcutaneous borders of both ulnae. This condition she had completely recovered from.

She was of somewhat massive build and led an active life, playing croquet and tennis and dancing vigorously. She came of a family which might be described as 'neurotic', though she herself displayed little or nothing of this tendency. Her digestion was good, and she was not subject to constipation or gastro-intestinal disturbance.

I was struck by the similar appearance of her fingers to that of Case I, both as regards the diffuse swelling and the nodules. She too had been told that the condition was incurable and would inevitably lead to painful crippling of her hands, a prognosis which distressed her greatly as she earned a small income by practising and teaching lacemaking. Her blood examination showed no abnormality except a marked delay in the coagulation time during the attacks.

Case III. Elsie M., aged 11, a slightly-built girl with dark auburn hair and a freckled skin, had complained for three years of pains in the joints for which on several occasions she had been admitted to the Bristol Royal Infirmary with the diagnosis of subacute rheumatism. The case was singular from the fact that although I had seen her in the wards each time she was admitted, I could never discover any swollen joints, nor indeed anything the matter with her.

One night, however, in April 1910, her mother telephoned and asked if I would see her, and she came to my house at 9.30 p.m. with numerous nodules on her knees, elbows, and fingers. On the knees and elbows tbese nodules were cutaneous and subcutaneous, about the size of hazel nuts, fairly hard but pitting on firm pressure with a slightly dusky red tinge in the overlying skin. They were very painful, making movement stiff and difficult. Several nodules appeared to be attached to both patellar tendons. In the fingers there were deep-seated nodules about the size of peas, looking and feeling like ganglions on the tendon sheaths; there was no dullness or oedema of the skin. There were the same spindle swellings between the finger-joints with the contractions at the joints, which had attracted attention in the two previous cases. The swellings were said to rise about 6 p.m. every night and disappear by the morning. They were most sovere when the child was fatigued by physical exertion.

Sometimes a particularly bad outburst was preceded by an attack of vomiting with or without diarmoea. In October, 1910, she was admitted to the Bristol Royal Infirmary suffering from jaundice, apparently of the catarrbal type, at the onset of which there was severe vomiting. During ber stay in the ward on this occasion the evening attacks of urticaria tuberosa occurred frequently. The patient showed a marked degree of hyper-extension of the fingers at all jointsa feature which was also noticeable in Case II, and serves to accentuate the difference between this condition and the deformities usually met with in rheumatoid arthritis. The delayed coagulation time of the blood during the attacks was pronounced.

There was no sign of organic disease, and the heart remained unaffected in spite of repeated attacks of so-called subacute and nodular rheumatism.

The attacks still continue, and are not controlled by administration of calcium salts; the patient regards them philosophically and is now very little disturbed by them. 
Case IV. C. S., aged 17, a short, stout girl with a fresh ruddy complexion, whom I was asked to see early in April, 1911, with Dr. Falconar at Shirehampton.

She had been ill for six days; the attack commenced suddenly with headache, frequent violent vomiting with diarrhoea, and pains in the chest and joints, the latter becoming swollen. The temperature had on one occasion risen to $99^{\circ} \mathrm{F}$. The joint pains were so severe that morphia was resorted to after failure of salicylate and other remedies.

Nine years ago the patient had a severe attack of diphtheria, after which attacks similar to the one described appeared to follow. The administration of antitoxin is probable as the child was treated in the City Fever Hospital. These attacks come on at frequent intervals, they are often accompanied by vomiting, and also signalized by painful swollen joints. Some attacks are so slight that the patient is not confined to bed. They usually come on at nights and last till the morning. Moderate movement brings relief, but a long walk or fatigue precipitates or aggravates the attack. This patient had sought relief at Bath four years previously, and after leaving Bath suffered from an attack of rheumatic fever (so called) for several weeks. She has also had influenza several times.

When I saw her she was lying in bed looking plump and rosy-cheeked and not ill, but movement caused her great pain. Her temperature was 98.4 , the pulse rate 84 , and the respiration rate 24 .

She complained of great pain in both pectoral muscles and in the deltoids, which were extremely tender on pressure or movement; there was also pain over the scapulae and rhomboids, but there was no swelling, and no nodules were discovered in these regions.

The right elbow was enlarged by an ill-defined fluctuating swelling just external to the olecranon and not communicating with the joint. The fingers of both hands were stiff but without effusion into any joints and without nodules; the swelling was situated between the joints with constriction at the joints.

Both legs were mottled and swollen resembling a fading nettle-rash, but there had been no itching. About the middle of the left calf were two discrete deep-seated painful lumps about $1 \frac{1}{2}$ inches in diameter which felt in shape and consistency like soft ginger-nut biscuits. The skin over them was not reddened or infiltrated, and the nodules were apparently attached to muscle or deep fascia. They were tender and pitted on prolonged pressure, but there was no pitting of the skin. None of the joints of the lower limb was swollen. Three similar but smaller nodules were present in the calf of the right leg. In three days the condition had cleared up, but during the rest of the month of April, after admission to the Royal Infirmary, she had at intervals of two or three days attacks of severe pain, sometimes, not always, accompanied by localized swellings, and of a transient character.

About a month after leaving the Royal Infirmary the girl came to see me with a swelling over the left ankle, a typical patch of deep-seated oedema about $1 \frac{1}{2}$ inches in diameter, and she told me that she had had a similar swelling in the thigh pointing to the inner condyle of the femur about the inner tuberosity.

Her attacks were less severe and less frequent after this, possibly because she was more careful in her diet and regulated her bowels with magnesia.

Unhappily she died in 1912 of cerebral complications following an acute suppurative otitis media. No post-mortem examination was allowed.

Case V. Mrs. S., aged 27, the wife of a medical man with whom I was well acquainted and whose children. I had frequently attended for lichen urticatus, and many curious urticarial rashes.

She had suffered for neally two years from some form of chronic rheumatism which she had been warned would lead to crippling deformities of the joints. 
I had never been consulted by her for her own health. One day, however, I was seeing the children for one of their numerous urticarial outbursts, and she held out her hand saying she wished I could cure her fingers which were crippled with rheumatism. But the fingers were not swollen at the joints, there was instead a constriction at each joint and spindle-shaped swellings between. There were two or three nodules the size of peas apparently situated in or on the extensor tendon sheaths at a distance from the joints. On inquiry, I found that these were the nodes of the rheumatoid arthritis from which such dire results were apprehended. These nodules were sometimes attended by painful lumps and stiffiness in the small of the back and gluteal regions.

I have seen her on many occasions since suffering from the deep-seated nodosities of urticaria tuberosa, and at rarer intervals from spontaneous bruising, but she has never developed a nettle-rash.

Her mother is the subject of most severe nettle-rashes.

\section{Blood-coagulation Times.}

By Addis and Sabrazé's method. Temperature $18.5^{\circ} \mathrm{C}$.

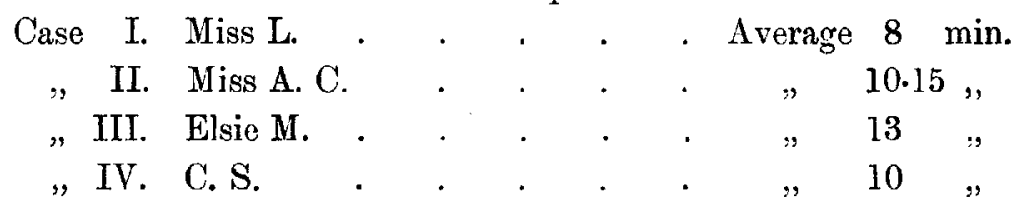

Control (Egomet) varies from 6.5 to 7 minutes by the same method and taken with each patient.

\section{Treatment.}

The essential point to recognize in the treatment of this condition is its urticarial nature, and, moreover, to realize the exceptional disposition which its victims display towards developing drug eruptions.

The salicylate group, colchicum, and the iodides are useless; the latter frequently adds to their miseries. So, too, do the bromides and chloral and the many hypnotic drugs which are apt to produce exudative erythematous rashes. In the majority of attacks which $I$ have seen in the cases described the speediest and greatest relief has been obtained by prompt and free purgation, a dose of calomel or blue pill followed by sulphate of magnesia two or three times a day sufficient to produce one drastic purge and one or two loose daily actions of the bowels is the only satisfactory treatment I know.

Regular administration of calcium nay ward off the attacks, but it does not appear to cut them short in the same fashion as the other alkaline earth, magnesia. It has the disadvantage of tending towards constipation.

Diet is of the utmost importance. My own experience has been that each patient subject to urticaria (of any type) is a law unto himself, and each case must be carefully studied until by varying exclusions from the diet an optimum is reached, where the attacks are reduced to their lowest frequency, Speaking generally, the plainest diet is best; milk alone during an attack is to be recommended, but some of my patients have told me that the surest plan for curing an attack of urticaria is to starve it out and live for a day or so after the outbreak on nothing but bread and butter or toast, and water. An heroic remedy, but one based on sound experience and much suffering. 


\section{Conclusions.}

In conclusion, one may say that the treatment of urticaria tuberosa like that of other forms of urticaria is unsatisfactory, but its correct diagnosis is of the utmost importance. A patient who is subject to attacks of urticaria tuberosa derives no little comfort and relief from the knowledge of the nature of the disease, and the reassurance that it brings no permanent disabilities in its wake. The minor discomforts of a nettle-rash may be laughed away, but the gloomy anticipation of helpless deformity from rheumatoid arthritis may spoil a life long ere the crippling has come.

The pathology and histology of these nodules cannot be proved by direct examination, as the patients are not as a rule agreeable to deep incisions into muscles and tendon sheaths for the purpose of removing tissues for microscopical examination. They are the less inclined to these methods of research from their experience of the transitory nature of the swellings.

The behaviour of the swellings leaves no room for doubt that they are of the nature of urticarial effusions or angeio-neurotic oedema.

It is regrettable that in Quincke's survey of angeio-neurotic oedemas this nodular form seems to have been lost sight of. Since rheumatoid arthritis claims Heberden's nodes, Haygarth's nodes, and osteo-arthritis, the easiest explanation of these cases of urticaria tuberosa was to throw them into the same group. Then with inappropriate remedies and a discouraging prognosis to leave the sufferer to discover that Fate was not as hard as she was painted, in fact that urticaria tuberosa was a disease differing totally in its effects and sequels from arthritis deformans, to which superficially it bears so close a resemblance.

Evidently a group exists, recognized by Willan, in which the urticaria effusion is so limited that it does not present the characteristic features of either an urticaria or angeio-neurotic oedema.

\section{BIBLIOGRAPHY.}

1. Frank, J. P., De Curandis Hominum Morbis, 1792, iii. 108.

2. Willan, On Cutaneous Diseases, 1808, 428.

3. Cazenave, Nouvelle Bibliothèque médicale, 1827, 62 (Bull. de l'Athénée).

4. Bateman, T., Cutaneous Diseases, Lond., 1829, 7th edit., 138.

5. Rayer, P., Diseases of the Skin, Lond., 1835, 197.

6. Demarquay, Gaz. des Hópitaux, Paris, 1842, xv. 165.

7. Graves, Clinical Medicine, 1843 (Syd. Soc., edit. 1884. i. 513).

8. Speyer, Deutsche Klinik, 1853, v. 268.

9. Fouquet, Berl. klin. Woch., 1865, ii. 327.

10. Baker, Med.-Chiv. Trans., Lond., 1881, lxiv. 289.

11. Dubreuilh, Gaz. drs Hópitaux, Paris, 1892, lxv, 1133, 1337.

12. Collins, Amer. Journ. Med. Sci., 1892, N. S., civ. 654.

13. Osler, Osler and MoCrae, System of Medicine, Lond., 1909, vi. 657.

14. Garrod, Allbutt and Rolleston, ibid., 1907, iii. 14.

15. Batten, ibid., 1910, vii. 7.

16. Anderson, Treatise on Diseases of the Skin, Lond., 1887, 220. 